\title{
Computerized tools in psychology: cross cultural and genetically informative studies of memory
}

\author{
V. Ismatullina ${ }^{1}$, I. Zakharov ${ }^{1}$, E. Nikulchev ${ }^{2}$ and S. Malykh ${ }^{1, a}$ \\ ${ }^{1} \mathrm{Psychological}$ Institute of Russian Academy of Education, Laboratory of developmental behaviour genetics, 125009 Moscow, Russia \\ ${ }^{2}$ Moscow Technological Institute, 119334 Moscow, Russia
}

\begin{abstract}
In this article we presented the computerized tools for psychological studies of memory. The importance of implementing computerized automated tools for psychological studies is discussed. It has been shown that this tools can be used both for cross-cultural and genetically informative studies. The validity of these tools for cross-cultural and genetically informative studies of memory can be seen as the first step to use automated computerized tools for big data collection in psychology.
\end{abstract}

\section{Introduction}

In the modern era of managed care and socialized medicine, a pragmatic concern for practitioners is the extent to which computers can save them time and money. In the recent time period the use of online computerized in psychological research is growing fast. It is becoming standard practice to administer various forms of questionnaires via social networks and special websites (e.g., https://www.1ka.si). In addition, the development of touch-sensitive screens, touchpens, and voice-activated software allows examinees to make responses through a variety of input media. Advantages include increased efficiency, the achievement of highly standardized administrations, and automated response recording that would be difficult to accomplish by hand. For example, response times can be recorded with millisecond precision. There is also the possibility of adapting tests to suit the test-taker's ability level [1]. It has been shown, that performance scores is stable across different types of web-browsers and devices [2].

One example of computerized tool for psychological assessment is CANTAB [3]. Although the original aim of the CANTAB was to assess patterns of cognitive decline insubtypes of dementia in elderly participants, it has since been employed in clinical neuropsychological research across age groups. Performance has been characterized in a variety of psychiatric and neurological conditions, including attention deficit hyperactivity disorder [4], schizophrenia [5], affective disorders [6,7], anxiety disorders [8], Alzheimer's dementia [9] and Parkinson's disease [10]. Today it is of immense practical importanceto obtain baseline data on healthy samples who are representative of different age and ability levels to define a standard against which the performanceof various patient groups can be assessed.
It was empirically demonstrated that task performance is comparable in children who are native versus nonnative English speakers when English is the language used to present each task [11]. In general, children found the computerized testing format to be interesting and motivating. It is worth noting that it is not only the overall testing format that is suitable for children. Children can be tested on the same tests and using exactly the same item sets that are employed in adult studies. Thus, this battery has been successfully used, without modification, to measure cognitive functions in individuals from ages 4 to over 90 years. In 4-12-yearold children, internal consistency coefficients are uniformly high, ranging from .73 for a measure of reaction time latency to .95 [11].

The most current version of the battery is a Windowsbased program that operates on a PC platform and utilizes touch screen technology. The battery consists of 13 subtests that include measures of motor skill, visual attention, memory, and working memory. All task stimuli are nonverbal, consisting of geometric designs or simple shapes, and language proficiency is necessary only to understand the instructions prior to task initiation. The validity of the CANTAB for assessing brain-behavior relations in adults has been supported by numerous patients with brain lesions, degenerative disorders, and psychiatric illness $[12,13]$. The impacts of psychopharmacological treatments on performance have also been examined $[14,15]$.

Functional magnetic resonance imaging (fMRI) and positron emission tomography (PET) have provided information regarding the neural substrates underlying adults_performance on several tasks [16]. These findings indicate that the CANTAB is sensitive to the presence of brain dysfunction in adults and that discrimination among subtypes of brain disorder ispossible using profile

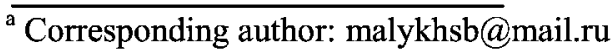


interpretation. It seems promising to use CANTAB tests together with brain activity recording, which are becoming widely available due to the progress in mass production of EEG headsets (e.g., https://emotiv.com/ epoc.php). In this article we want to present the use of computerized tools in cross cultural and genetically informative studies.

\section{Material and methods}

In our study, we used computerized tests of cognition, The Cambridge Neuropsychological Test Automated Battery [3]. CANTAB is relatively new to the field of quantitative genetic studies, using the accuracy and rigour of computerised psychological testing whilst also allowing for a wide range of ability avoiding ceiling and floor effects in the young [14] and old [17], respectively. As have been noted, the tests are culture and language free (and can even be administered without verbal instruction).

The testability of CANTAB in different samples has been showed in clinical and normal samples. At the same time there are only few studies dedicated to availability of using CANTAB tests in children population $[11,18]$. CANTAB has been reported to have high applicability in the evaluation of children. In 4-12-year-old children, internal consistency coefficients are uniformly high, rangingfrom .73 for a measure of reaction time latency to .95 for performance on the self-ordered search task. We showed that reliability of SWM and PRM preformancescoresreliabilityis .71-.73.

We used two CANTAB subtests, Spatial Working Memory and Pattern recognition Memory in several experiments [19, 20]. We used memory subtests as memory is one of the most important cognitive functions which ensures unity an dintegrity of human behavior. Memory consists of a number of different entities relying on different brain systems. Memory can be categorized according to the nature of the material memorized. Investigation of unique features of visual recognition and spatial working memory is particularly interesting due to the fact that it contributes to a broader understanding of memory mechanisms ingeneral.

All tasks were administered through the use of a touch-screen computer.

\subsection{Spatial Working memory test}

Spatial Working Memory (SWM) task is self-ordered serial search task, where the participants were shown a group of boxes on the computer screen. They were told that a token was hidden beneath one of the boxes on the screen and they had to search through the boxes to find it. Participants did this by touching a box to discover whether or not a token was hidden beneath it. After participants found the token, they were shown the same set of boxes and instructed to find the next token. Participants were told that once a token had been found under a particular box, the box would never again hide a token. In a set of trials, each box eventually had a hidden token beneath it. Thus, to perform this task with the fewest number of errors, the participants needed to remember which of the boxes had previously hidden a token within a set of trials. The number of boxes on the screen, under which the participants needed to find the tokens determined the level of working memory load. Either four, six, or eight boxes were presented on the screen at one time. As the memory load increased from four to six to eight boxes, the participants needed to remember whether or not a token had been previously located under a larger number of boxes and over a larger number of trials. After a participant had found every token in a set of boxes, both the color and position of the boxes changed to begin the next set of trials; the dimensions of the boxes did not change throughout the task. Each memory load condition (i.e., number of boxes on the screen) was presented four times, with the four 4box sets followed by the four 6-box sets and then the four 8 -box sets. Thus, a total of 72 tokens needed to be located in 12 search sets. To insure that participants understood the task, testing began with a practice set in which targets were hidden sequentially beneath three search boxes.

\subsection{Pattern recognition memory test}

Pattern Recognition Memory (PRM) is a test of visual pattern recognition memory in a 2-choice forced discrimination paradigm. This test is sensitive to dysfunction in medial temporal areas of the brain and relatively insensitive to dysfunction in the frontal lobe. The subject is presented with series of 12 visual patterns, one at a time, in the center of the screen. The patterns are designed so that they cannot easily be given verbal labels. In the recognition phase, the subject is required to choose between a pattern they have already seen and a novel pattern. In this phase, the test patterns are presented in there verse presentation. The sub-test is repeated with a new set of 12 patterns to be remembered.

\subsection{Statistical analysis}

In our study we used two approaches: cross-cultural and genetically informative designs. To compare the groups in cross-cultural approach we analyzed standard descriptive statistics and performed a univariate ANOVA.

The contributions of genetic and environmental influences on individual differences in cognitive abilities were estimated using the structural equation modeling [21]. The data from $M Z$ and $\mathrm{DZ}$ twins are used to estimate the contributions of the following components of phenotypic variance: additive (A) and non-additive (D) genetic factors, shared (C) and non-shared (E) environmental factors. The last component also includes the estimation of variance that comes from measurement error.

\section{Results}

\subsection{Experiment 1. Cross-cultural study}

3.1.1. SWM subtest. The study involved 289 adolescents, 172 of them were from Kyrgyzstan and 117 - from Russia (Mean age 12.9 years, SD=2,12 years).The participants were assessed using the SWM 
subtest. To compare the groups by country we performed a univariate ANOVA. There were no significant differences in means across countries (Between errors: $\mathrm{F}[1,287]=0.022, \quad \mathrm{p}=0.881 ; \quad$ Total errors: $\mathrm{F}[1,287]=0.038, \mathrm{p}=0.846 ;$ Strategy $\mathrm{F}[1,287]=0.687$, $\mathrm{p}=0.408$; see also Table 1). It should be noted that means and standard deviations in Russian and Kyrgyz adolescents corresponded with the CANTAB norms.

3.1.2. PRM subtest. The study involved 343 adolescents, 223 of them were from Kyrgyzstan and 120 - from Russia (Meanage 12.9 years, $\mathrm{SD}=2,19$ years).
To compare the groups by country we performed a univariate ANOVA. There were no significant differences in means across countries, but there were difference in the number of correct answers. Though it was significant, the effect size of 1 correct answer is negligible (Meanlatency: $\mathrm{F}[1,343]=0.778, \quad \mathrm{p}=0.378$; Correctanwsers: $\quad \mathrm{F}[1,343]=8.195, \quad \mathrm{p}=.023$; see also Table 2).

It should be noted that means and standard deviations in Russian and Kyrgyz adolescents corresponded with the CANTAB norms.

Table 1. Descriptive statistics for SWM test.

\begin{tabular}{|l|l|l|l|l|l|}
\hline \multicolumn{2}{|c|}{} & \multicolumn{1}{|c|}{ Mean } & \multicolumn{1}{c|}{ SD } & \multicolumn{1}{c|}{ SE } & \multicolumn{2}{c|}{$95 \%$ CI } \\
\hline \multirow{3}{*}{ Betweenerrors } & Kyrgyz & 38.86 & 19.496 & 1.487 & {$\left[\begin{array}{ll}35.93, & 41.79\end{array}\right]$} \\
\cline { 2 - 6 } & Russian & 38.52 & 18.025 & 1.666 & {$\left[\begin{array}{ll}35.22, & 41.82\end{array}\right]$} \\
\hline \multirow{3}{*}{ Strategy } & Kyrgyz & 35.09 & 5.057 & 0.386 & {$\left[\begin{array}{ll}34.33, & 35.85\end{array}\right]$} \\
\cline { 2 - 6 } & Russian & 35.54 & 3.659 & 0.338 & {$\left[\begin{array}{ll}34.87, & 36.21\end{array}\right]$} \\
\hline \multirow{2}{*}{ Totalerrors } & Kyrgyz & 39.50 & 19.799 & 1.510 & {$\left[\begin{array}{ll}36.52, & 42.48\end{array}\right]$} \\
\cline { 2 - 6 } & Russian & 39.05 & 18.288 & 1.691 & {$\left[\begin{array}{ll}35.70, & 42.40\end{array}\right]$} \\
\hline
\end{tabular}

Table 2. Descriptive statistics for PRM test.

\begin{tabular}{|l|l|c|c|c|c|}
\hline \multicolumn{2}{|c|}{} & Mean & SD & SE & $95 \%$ CI \\
\hline \multirow{3}{*}{ Mean latency } & Kyrgyz & 1970.39 & 480,56 & 33.35 & {$\left[\begin{array}{ll}35.93, & 41.79\end{array}\right]$} \\
\cline { 2 - 6 } & Russian & 1920.57 & 470.43 & 45.57 & {$\left[\begin{array}{ll}35.22, & 41.82\end{array}\right]$} \\
\hline \multirow{2}{*}{$\begin{array}{l}\text { Correct } \\
\text { anwsers }\end{array}$} & Kyrgyz & 20.63 & 2.57 & 0.168 & {$\left[\begin{array}{ll}34.33, & 35.85\end{array}\right]$} \\
\cline { 2 - 6 } & Russian & 21.45 & 2.42 & 0.230 & {$\left[\begin{array}{ll}34.87, & 36.21\end{array}\right]$} \\
\hline
\end{tabular}

\subsection{Experiment 2. Genetically informative study}

Previously, different authors reported the results of research in specific cognitive abilities such as processing speed, working memory, spatial ability and verbal ability in which have shown heritabilities that range from $30 \%$ to $60 \%$ in both young and elderly subjects. Such studies have employed the cognitive tests such as the 'pencil and paper' tests used in the Hawaii Family Study of Cognition [22] and 'Working Memory Span' tasks [23]. However, these testing procedures of testing doesn't allow for studying the pure cognitive component of the task [24]. In a study of executive functions, Friedman [25] showed that $31-71 \%$ of individual differences can be explained by genetic factors, while the environmental impact range from 50 to $71 \%$.

3.2.1. SWM subtest. Our genetically informative sample for SWM subtest consisted of 688 adolescences (339 twin pairs, $M Z=118, D Z=143$ ) aged from 10 to 16 years (mean age 12.99, SD 2.18).

Cross-twin correlations of "Between errors" WM measure were 0.592 for $\mathrm{MZ}$ and 0.352 for $\mathrm{DZ}$. The individual differences in "Between errors" measure were explained by additive genetic $(A=60 \%)$ and non-shared environmental $(E=40 \%)$ effects. For "Total errors" parameter the cross-twin correlations were 0.594 for $\mathrm{MZ}$ and 0.350 for DZ. Most suitable model was also genetic $(\mathrm{A}=60 \%, \mathrm{E}=40 \%$ ). "Mean time to last response 8 boxes" measure was explained by shared environmental
$(C=41 \%)$, and non-shared environmental $(59 \%)$ effects. Cross-twin correlations for "Strategy" measure were 0.406 for $\mathrm{MZ}$ and 0.151 for DZ. For this parameter most suitable model was additive genetic model where $\mathrm{A}=$ $39 \%$ and $\mathrm{E}=61 \%$.

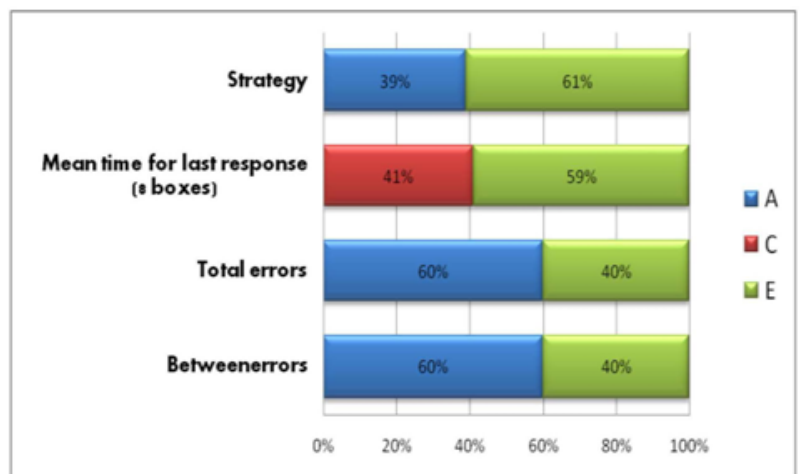

Figure 1. Parameter Estimates for Additive Heredity (A), Common Environment (C), and Unique Environmental Influences (E) by Equation Modeling of SWM test

MZ co-twin correlations were higher than DZ co-twin correlations for most variables. All variables were tested for assumption of ACE estimation. After testing "Within errors", "Double errors", "Mean time to first response (8 boxes)" parameters were excluded from model fitting test.

3.2.2. PRM subtest. The PRM sample included 339 twins pairs (118 MZ, $221 \mathrm{DZ})$, aged from 10 to 17 years (mean age 13, SD 2.19). 
Cross-twin correlations of "Mean correct latency" PRM measure were 0.39 for $\mathrm{MZ}$ and 0.14 for DZ. The individual differences in "Mean correct latency" measure were explained by additive genetic $(\mathrm{A}=37 \%)$ and nonshared environmental $(E=63 \%)$ effects. For "Number correct" parameter the cross-twin correlations were 0.33 for $\mathrm{MZ}$ and 0.29 for DZ. Most suitable model was full model where $\mathrm{A}=13 \%, \mathrm{C}=22 \%$, and $\mathrm{E}=64 \%$.

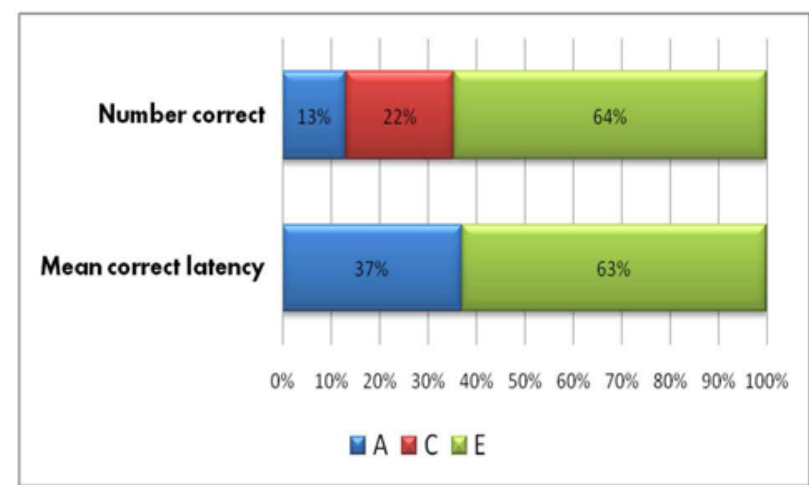

Figure 2. Parameter Estimates for Additive Heredity (A), Common Environment (C), and Unique Environmental Influences (E) by Equation Modeling of PRM test

Thus, different measures of pattern recognition memory were associated with additive genetic and common environment factors with substantial impact of non-shared environment.

\section{Conclusions}

It is important for psychological studies today to keep up with current trends such as using big data for research. Computerized tools provide incredible source of information given wide prevalence of social media and web-site in everyday people life. It gives unique chance to enlarge study samples that can be invaluable for such areas as cross cultural or genetically informative studies $[27,28]$. We showed that CANTAB is sutiable for that purpose. CANTAB, like other computerized tests, has limitations. It may be important for practitioners, in formulating treatment to facilitate the collection and storage of vast amounts of normative developmental and clinical data, that can lead to new hypotheses and knowledge regarding cross cultural, genetic, and environmental sources of individual differences.

\section{References}

1. B.L. Roper, Y.S. Ben-Porath and J.N. Butcher, Journal of Personality Assessment, 65, 358 (1995)

2. A. Chetverikov and P. Upravitelev. Behavior Research Methods (2015)

3. E. Strauss, E.M.S Sheman and O. Spreen, $A$ Compendium of Neuropsychological Tests: Administration, Norms, and Commentary (3rd. Ed) (Oxford University Press, 2006)

4. T.W. Robbins, M.A. Mehta and B.J. Sahakian. Science, 290, 2275 (2000)
5. R. Elliott, P.J. McKenna, T.W. Robbins and B.J. Sahakian, Psychological Medicine, 25, 619 (1995)

6. B.C. Beats, B.J. Sahakian and R. Levy. Psychological Medicine, 26, 591 (1996)

7. R. Elliott, S.C. Baker, R.D. Rogers, D.A. O'leary, E.S. Paykel, C.D. Frith, R.J. Dolan, and B.J. Sahakian. Psychological Medicine, 27, 931 (1997)

8. D.M. Veale, B.J. Sahakian, A.M. Owen and I.M. Marks, Psychological Medicine, 26, 1261 (1996)

9. K.W. Lange, B. J. Sahakian, N.P. Quinn, C.D. Marsden and T. W. Robbins. Journal of Neurology, Neurosurgery \& Psychiatry, 58, 598 (1995)

10. A.M. Owen, J.L Iddon, J.R. Hodges, B.A. Summers and T.W. Robbins, Neuropsychologia, 35, 519 (1997)

11. M. Luciana and C.A. Nelson, Developmental Neuropsychology, 22, 595 (2002)

12. K.S. Fowler, M.M. Saling, E.L. Conway, J.M. Semple and W.J. Louis, Journal of the International Neuropsychological Society, 3, 139 (1997)

13. S. Rahman, T.W. Robbins and B.J. Sahakian, Dementia and Geriatric Cognitive Disorders, 10, 15 (1999)

14. J.T. Coull, H.C. Middleton, T.W. Robbins and B.J. Sahakian, Psychopharmacology, 120, 322 (1995)

15. R. Elliott, B.J. Sahakian, K. Matthews, A. Bannerjea, J. Rimmer and T. W. Robbins. Psychopharmacology, 131, 196 (1997)

16. A.C.H. Lee, A.M. Owen, R.D. Rogers, B.J. Sahakian and T.W. Robbins, Utility of CANTAB in functional neuroimaging, pp. 366-378 (2000)

17. B.J. Sahakian, R.G. Morris, J.L. Evenden, A. Heald, R. Levy, M. Philpot and T.W. Robbins, Brain, 111, 695 (1988)

18. C.R. De Luca, S.J. Wood, V. Anderson, J. Buchanan, T. M. Proffitt, K. Mahony and C. Pantelis, Journal of Clinical and Experimental Neuropsychology, 25, 242 (2003)

19. V. Ismatullina, I. Voronin, A. Shelemetieva and S. Malykh, Procedia - Social and Behavioral Science, 146, 353 (2014)

20. Y. Davydova, V. Ismatullina, I. Voronin, O. Ovcharova, E. Sabirova and S. Malykh, Procedia Social and Behavioral Sciences, 86, 419 (2013)

21. M. Neale and L. Cardon. Methodology for Genetic Studies of Twins and Families (Springer, 1992)

22. J.C. DeFries, R.C. Johnson, A.R. Kuse, G.E. McClearn, J. Polovina, S.G. Vandenberg and J.R. Wilson, Behavior Genetics, 9, 23 (1979)

23. M.K. Healey and A. Miyake, Quarterly Journal of Experimental Psychology, 62, 733 (2006)

24. P. Schatz and J. Browndyke, The Journal of head trauma rehabilitation, 17, 395 (2002)

25. N.P. Friedman, A. Miyake, S.E. Young, J.C. DeFries, R.P. Corley and J.K. Hewitt, Journal of Experimental Psychology: General, 137, 201 (2008)

26. I. Zakharov, V. Ismatullina and S Malykh, Theoretical and Experimental Psychology, 7, 79 (2014)

27. I. Zakharov, V. Ismatullina and I. Voronin, Theoretical and Experimental Psychology, 8, in press. 